\title{
Treatment of familial pulmonary arterial hypertension with novel nebulizer (BreelibTM) for iloprost inhalations - first use outside of clinical trials
}

\author{
Katarzyna Łabuś ${ }^{1 *}$ and Agnieszka Pawlak ${ }^{2}$ \\ ${ }^{1}$ Clinical Hospital of the Ministry of Internal Affairs, Department of Invasive Cardiology, Warsaw, Poland \\ ${ }^{2}$ Department of Applied Physiology, Mossakowski Medical Research Centre, Polish Academy of Sciences, Warsaw, Poland
}

\section{Opinion}

A 23 year old female was admitted to The Invasive Cardiology Department in October 2013 with suspicion of pulmonary arterial hypertension (PAH) estimated by transthoracic echocardiography.18 and 34 year old cousins of the patient suffers from idiopathic PAH and another one died in age of 14 from $\mathrm{PAH}$, what suggested familial PAH.

On admission patient presented NYHA (New York Heart Association) class III. Echocardiography revealed enlarged right ventricle (RV-47 mm), dilated pulmonary trunk, paradoxical motion of intraventricular septum, D-shaped left ventricle, pulmonary and tricuspid insufficiency with estimated RV systolic pressure (RVSP) $46 \mathrm{mmHg}$ and mean pulmonary arterial pressure (mPAP) $34 \mathrm{mmHg}$. In the right heart catheterization (RHC) the following values were measured: RV-59/12(15) mmHg, PAP-56/45(51) mmHg, pulmonary capillary wedge pressure (PCWP) $-7 \mathrm{mmHg}$, cardiac output-4,95 1/min, cardiac index-2,86 1/min/m2, PVR-15,3 Woods Units. Vasoreactivity test with iloprost reaveled negative response (PAP - 57/40(48) $\mathrm{mmHg}$, PCWP-7 mmHg). The patient was qualified to treatment with Sildenafil (60 mg/day).

In January 2014 patient checked herself into outpatient's department in the $3^{\text {th }}$ week of pregnancy. Woman was informed of the extensive risk to her and her fetus.

During 30 weeks of pregnancy she had an estimated in echocardiography PAPs on $70 \mathrm{mmHg}$ and $\mathrm{mPAP}$ on $57 \mathrm{mmHg}$. The iloprost (6 inhalations/day) was added to sildenafil.

In $35^{\text {th }} \mathrm{hbd}$ patient's state was moderate with symptomps in NYHA class IV. Cesarean section at 35-week gestation was decided, with subarachnoid analgesia. After uncomplicated newborn (APGAR score of 10 , birth weight $2400 \mathrm{~g}$, crown-rump length $49 \mathrm{~cm}$ ) and placenta delivery, bradycardia $(20 \mathrm{bpm})$ and hypotonia with nondetectable blood pressure occurred. Finally short sudden cardiac arrest was observed. Hemodynamic stability was recovered by ephedrine and atropine administration. During the following days patient's condition was stable. PAH therapy (sildenafil, iloprost) was continued.

Copyright: @ 02018 Łabuś K. This is an open-access article distributed under the terms of the Creative Commons Attribution License, which permits unrestricted use, distribution, and reproduction in any medium, provided the original author and source are credited.
Three-year follow-up shows that the patient's state was stable with NYHA class I.

Echocardiographic examination (Figure 1) displayed PAP 34 $\mathrm{mmHg}$. The PAH therapy consists of sildenafil $(60 \mathrm{mg} /$ day $)$ and iloprost (8 inhalation/day- vial $20 \mathrm{mcg} / \mathrm{dL}$ ). In April 2017 she started to use Breelib, the new nebulizer for iloprost as a first patient outside clinical trials. The Breelib nebulizer was developed to provide a device specifically for iloprost inhalation, to reduce inhalation times and thereby to improve convenience for patients. After reduction iloprost dose (from 20 to $10 \mathrm{mcg} / \mathrm{mL} /$ inhalation) clinical condition improved. The Breelib nebulizer offers better absorption of iloprost in lungs and good tolerability. Just after switch she presented headache, redness of the face, without significant decrease of blood pressure.

When initiation of therapy with Breelib ${ }^{\mathrm{TM}}$ is considered in patients treated previously with iNeb ${ }^{\mathrm{TM}}$ nebulizer initial dose of iloprost should be reduced and subsequently increased only in case to ensure the good tolerance.

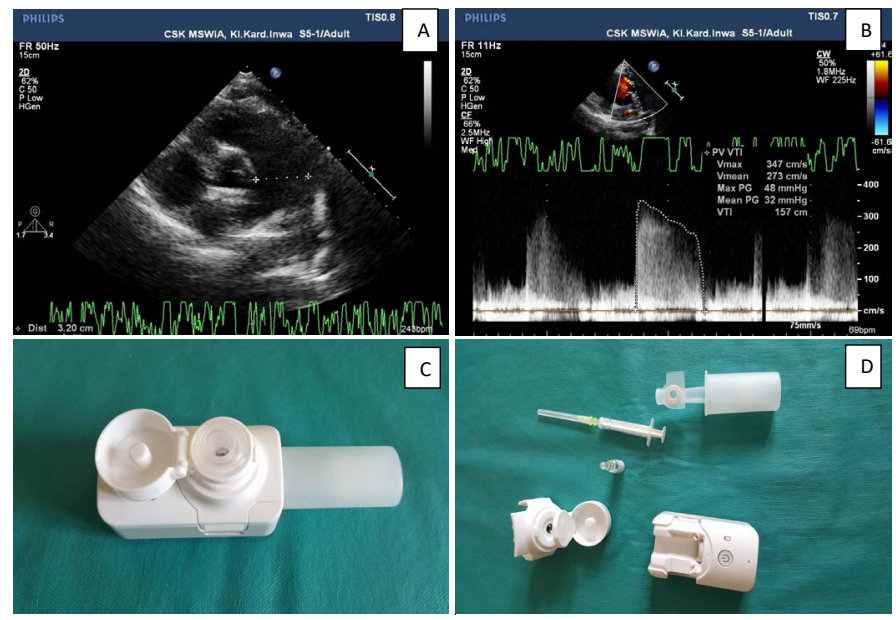

Figure 1. Echocardiographic assessment of pulmonary hypertension: A. enlargment of pulmonary trunk, B. Estimated pressure in pulmonary trunk, C\&D Breelib nebulizer

${ }^{\star}$ Correspondence to: Katarzyna Łabuś, Clinical Hospital of the Ministry of Internal Affairs, Department of Invasive Cardiology, Warsaw, Poland, E-mail: katelabus@gmail.com

Received: July 25, 2018; Accepted: August 23, 2018; Published: August 27, 2018 